\title{
Foveo-macular retinitis, solar retinopathy, and trauma
}

\author{
R. H. B. GREY \\ From the Department of Clinical Ophthalmology, Institute of Ophthalmology, and Moorfields Eye \\ Hospital, London
}

SUMMARY Three patients are described with foveal lesions resembling minute holes following trauma. The similarity of the lesions to foveomacular retinitis and solar retinopathy suggests that all these conditions produce a similar, localised neuroretinal lesion with sparing of the pigment epithelium. Loss of the photoreceptors at the fovea would be expected to produce a lesion resembling a small retinal hole.

Cordes (1944) described a series of patients who presented with blurred vision and a circular greyish lesion at the macula. After 1 to 2 weeks the lesion resolved, leaving a minute red foveal hole with variable visual recovery. He called the condition foveomacular retinitis. Subsequent authors considered this disorder to be central serous retinopathy, but Kerr and Little (1966) described a further series of patients with foveomacular retinitis and emphasised the difference between these two conditions. On account of the vast preponderance of young, fit, male military recruits in all the reported series of foveomacular retinitis and because of the similarity of the lesions to solar retinopathy, Ewald and Ritchey (1970) looked specifically for evidence of sun-gazing. Of their 47 patients $69 \%$ admitted to sun-gazing with or without the use of drugs, and they concluded that foveomacular retinitis was a self-inflicted condition largely to avoid active military service. Marlor et al. (1973) examined 147 Marine recruits with foveomacular retinitis in an attempt to elucidate the aetiology of this condition; they were unable to confirm sun-gazing as the cause.

The purpose of this report is to describe 3 patients with the appearance of foveomacular retinitis in whom the cause is known to be blunt trauma and to compare the fundus lesion with solar retinopathy.

\section{Case histories}

\section{CASE 1}

A 19-year-old male presented to the casualty department with blurred vision in the left eye after being hit by a football the previous day. The left visual acuity was $6 / 9$ and there was a mild anterior

Address for reprints: R. H. B. Grey, FRCS, Moorfields Eye Hospital, City Road, London ECIV 2PD uveitis. The left retina showed commotio retinae of the upper temporal quadrant and of the macula. The commotio settled over the following week, and the visual acuity improved to $6 / 6$, but a minute red lesion remained in the retina adjacent to the fovea (Fig. 1). Fluorescein angiography showed no abnormality.

Follow-up 5 months later showed the fundus to be unchanged.

\section{CASE 2}

A 36-year-old man was struck in the right eye by a metal band securing a packing case. He noticed

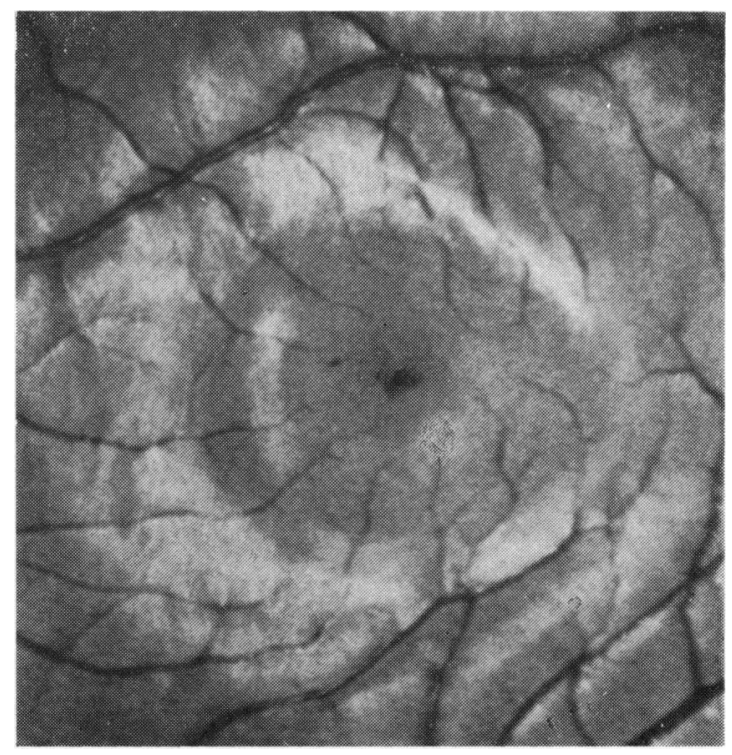

Fig. 1 Case 1: parafoveal lesion in left eye 


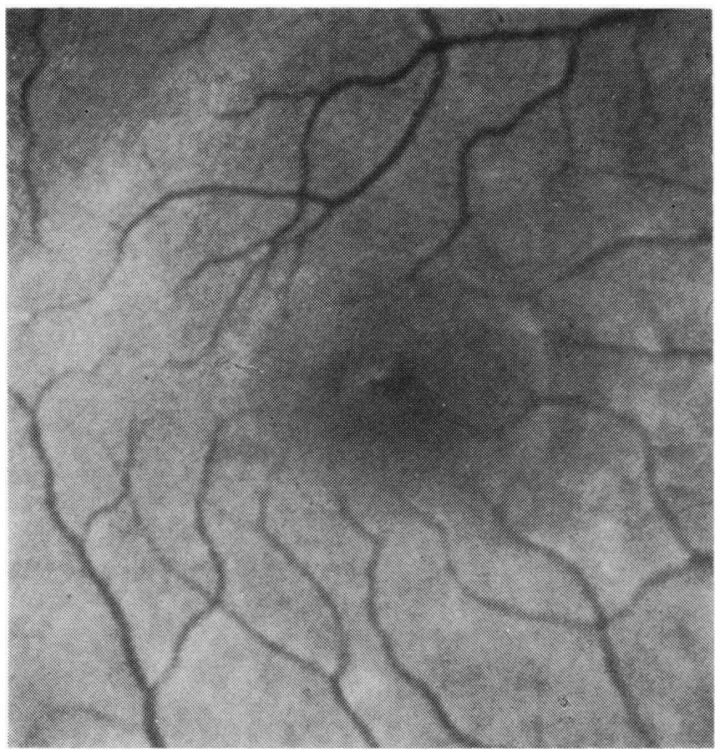

Fig. 2 Case 2: foveal lesion in right eye

distortion of the right vision, and his acuity was $6 / 9$ in this eye. At the macula a small cystic lesion was seen in the retina which contained haemorrhage. The haemorrhage resolved, but a small red lesion remained at the foveola which resembled a tiny macular hole (Fig. 2). Fluorescein angiography was normal.

Eighteen months later the fundus remained unchanged, and the visual acuity was $6 / 9$.

\section{CASE 3}

A boy of 15 had a bicycle accident in which he sustained a left black eye. A few weeks later he presented to hospital with diminished vision in the left eye. On examination his left visual acuity was 6/24 and he was seen to have a small lesion at the foveolar which resembled a small macular hole (Fig. 3). Fluorescein angiography showed a transmission defect corresponding to the clinically visible foveal lesion.

Three years later the visual acuity, fundus appearance, and fluorescein angiogram were unchanged.

\section{Solar retinopathy}

Since 197213 patients have been examined with a definite history of watching an eclipse or sun-gazing. All but 2 of these had fundal abnormalities similar to those described in the above 3 cases (Fig. 4). The lesions were approximately $100 \mu \mathrm{m}$ in diameter and resembled a small foveal hole. They were red, with a sharply demarcated outline, and were confined

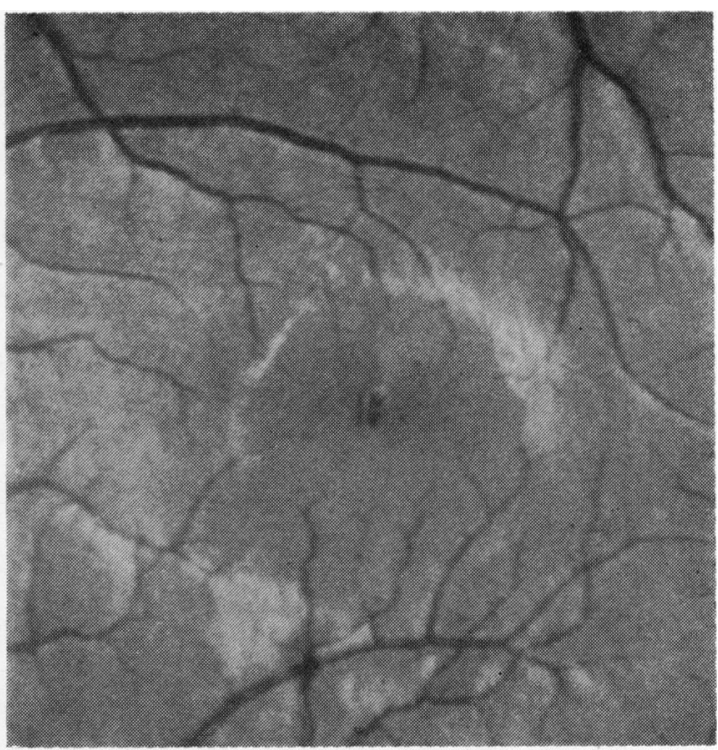

Fig. 3 Case 3: foveal lesion in left eye

entirely to the retina. The underlying pigment epithelium was normal. In 2 patients the lesions had been present for over 30 years with no apparent change in the features. The 2 atypical cases had diffuse pigment epithelial granularity at the fovea, with a redder than normal appearance; the retina appeared normal.

The visual recovery of these patients with solar

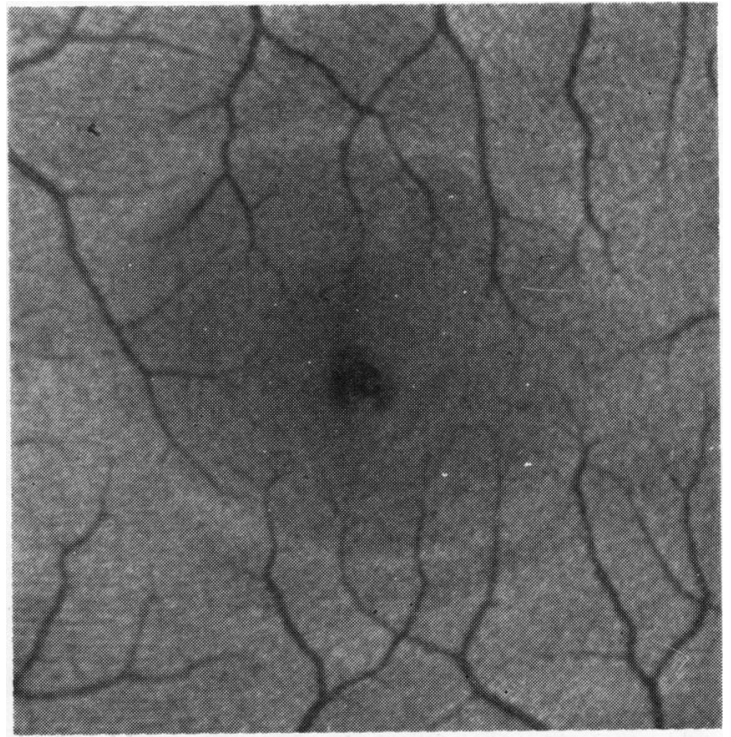

Fig. 4 Typical lesion of solar retinopathy after 2 years 
retinopathy was variable. Seven recovered to 69 or better, but 2 remained at $6 / 12,1$ at $6 / 18$, and 2 at 624 . One patient failed to re-attend.

\section{Discussion}

The lesions in the retinae of the 3 injured patients had the appearance of a macular hole similar to foveomacular retinitis (Cordes, 1944) and solar retinopathy (McCulloch, 1945; Rosen, 1948). Slitlamp biomicroscopy showed that there was thinning of the central neuroretina, though it was impossible to tell if the lesion was full thickness. The normal appearance of the retinal pigment epithelium on fluorescein angiography in Cases 1 and 2 suggested damage to the neuroretina giving rise to only partialthickness holes; the transmission defect in Case 3 possibly indicated a full-thickness hole or concomitant pigment epithelial damage.

At the fovea the retina consists almost exclusively of photoreceptors covered by the internal limiting lamina. Therefore selective destruction of the photoreceptors at the fovea would give the appearance of a retinal hole owing to the lack of retinal elements between the internal limiting lamina and the pigment epithelium. Diseases causing selective receptor loss without other changes would be expected to cause a similar foveal appearance whatever the aetiology.

Macular disease caused by sun-gazing may cause photocoagulation due to thermal injury (DukeElder, 1972) and result in damage to the retina and pigment epithelium (Penner and McNair, 1966; McFaul, 1969; Tso et al., 1975). However, light may produce photic damage to the retinal receptors alone (Tso t al., 1972; Clarke and Behrendt, 1972), and this is more likely watching an eclipse than looking straight at the sun (Geeraets et al., 1970). This form of selective damage may account for the characteristic appearance of a small macular hole, which is regularly reported in patients with solar retinopathy. Hatfield (1970) found 11 such cases in her review of 145 patients with eclipse burns, and Pang (1963) found a similar incidence with 2 out of 21 patients showing small holes. In the series described in the present study this appearance was particularly common, with 11 out of 13 patients affected in this way.

Trauma has been shown to cause receptor damage (Blight and Hart, 1977). In their experiments the damage was demonstrated at the site of impact and was not confined to the receptors; regeneration was also shown to occur. However, it is conceivable that selective photoreceptor damage may occur in man under some circumstances and produce the unique changes which give rise to the clinical appearance of a foveal red spot. Of the 3 patients described here with trauma only 1 was observed to have commotio retinae at the time of the injury, but the other 2 cases had injuries which could be considered to be serious enough to produce intraretinal oedema, although they were not seen at the time.

Any agent either physical or toxic which causes selective central photoreceptor loss would give rise to the appearance of foveomacular retinitis. During the course of this study 7 further patients were seen with foveal red spots. They had no history of ocular injury and denied sun-gazing; the aetiology of their foveomacular retinitis was obscure. One female patient dated her symptoms from her pregnancy, which was otherwise uncomplicated. Post partum her vision improved, and she was seen to have bilateral foveal red spots. Fluorescein angiograms were performed on all 7 of these patients; 5 were normal and 2 showed small transmission defects from the pigment epithelium and choriocapillaris underlying the lesions.

Foveomacular retinitis probably represents a small area of loss of the receptors in the retina, which has been shown to be caused by photic and physical injury. Kerr and Little (1966) and Marlor et al. (1973) suggested that on the basis of abnormal liver flocculation tests drug ingestion may be a possible cause. None of the patients described here had a history of drug taking.

I thank the surgeons of Moorfields Eye Hospital and $\mathrm{Mr} \mathrm{A}$. M. Hamilton for permission to publish their cases, Mr K. Sehmi for photographic help, and $\mathrm{Mr} \mathrm{A}$. C. Bird and Miss Heather Lucas for assistance with the manuscript.

\section{References}

Blight, R., and Dean Hart, J. C. (1977). Structural changes in the outer retinal layers following blunt mechanical nonperforating trauma to the globe: an experimental study. British Journal of Opthalmology, 61, 573-587.

Clarke, A. M., and Behrendt, T. (1972). Solar retinitis and pupillary reaction. American Journal of Ophthalmology. 73, 700-703.

Cordes, F. C. (1944). A type of foveo macular retinitis observed in the US Navy. American Journal of Ophthalmology, 27, 803-816.

Duke-Elder (1972). System of Ophthalmology, vol. 14, Pt. 2, pp. 885-896. Kimpton: London.

Ewald, R. A., and Ritchey, C. L. (1970). Sungazing as the cause of foveo macular retinitis. American Journal of Ophthalmology, 70, 491-497.

Geeraets, W. J., Nooney, T. W., Svoboda, J. R., and Ching, F. C. (1970). Solar Retinopathy following the eclipse of March 7 1970. Medical College of Virginia Quarterly, 6, 3-7.

Hatfield, E. M. (1970). Eye injuries and solar eclipse. Sight Saving Review, 40, 79-85.

Kerr, L. M., and Little, H. L. (1966). Foveo macular retinitis. Archives of Ophthalmology, 76, 498-504.

Marlor, R. L., Blais, B. R., Preston, F. R., and Boydon, 
D. G. (1973). Foveo macular retinitis; an important problem in military medicine: epidemiology. Investigative Ophthalmology, 12, 5-16.

McCulloch, C. (1945). Changes at the macula due to solar radiation. American Journal of Ophthalmology, 28, 11151122.

MacFaul, P. A. (1969). Visual prognosis after solar retinopathology. British Journal of Ophthalmology, 53, 534-541.

Pang, H. G. (1963). Eclipse retinopathy. American Journal of Ophthalmology, 55, 383-384.
Penner, R., and McNair, J. N. (1966). Eclipse blindness. American Journal of Ophthalmology, 61, 1452-1457.

Rosen, E. (1948). Solar retinitis. British Journal of Ophthalmology, 32, 23-35.

Tso, M. O. M., Fine, B. S., and Zimmerman, L. E. (1972). Photic maculopathy produced by the indirect ophthalmoscope. American Journal of Ophthalmology, 73, 686-699.

Tso, M. O. M., and La Piana, F. G. (1975). The human fovea after sungazing. Transactions of American Academy of Ophthalmology and Otolaryngology, 79, 788-795. 